\title{
Diagnosis of Enigmatic Non-Odontogenic Bony Pathology-Do Endodontist Have Any Role?
}

\author{
Nimisha $S^{1 *}$, Meghna $K^{2}$ and Ankit $A^{3}$ \\ ${ }^{1}$ Professor and Head of the Department, KM Shah Dental College and Hospital, Sumandeep \\ Vidyapeeth, India \\ ${ }^{2}$ Post graduate student, KM Shah Dental College and Hospital, Sumandeep Vidyapeeth, India \\ ${ }^{3}$ Reader, KM Shah Dental College and Hospital, Sumandeep Vidyapeeth, India
}

\section{Case Report}

Volume 5 Issue 2

Received Date: April 20, 2020

Published Date: May 11, 2020

DOI: $10.23880 /$ oajds-16000253

*Corresponding author: Nimisha Shah, Professor and Head of the Department, KM Shah

Dental College and Hospital, Sumandeep Vidyapeeth, Vadodara, Gujarat, India, Tel: +91 9998994299; Email: nshah7873@gmail. com

\section{Abstract}

The oral cavity is a reflection of our body. Pain in oro-facial region is complex, distressing condition which frequently overlaps with various surgical and medical disciplines. Accurate diagnosis may turn out to be challenging for experienced clinician as well and Non-odontogenic toothache is one such entity. A misdiagnosis can lead to unnecessary treatment for the patient. One of such entity is osteomyelitis having radiographic similarity with periapical radiolucency during its initial stage. Often such situation can lead to inaccurate diagnosis during dormant stage. Though due to advancement in medicine the incidence of osteomyelitis had a great decline still it can be an enigma to an endodontist in diagnosing the condition. We hereby report one such condition encountered in the department of Conservative dentistry and emphasizes on a multidisciplinary approach, highlighting the importance endodontist in diagnosis of an rare entity osteomyelitis its features and diagnosing such situation during our routine clinical practice.

Keywords: Oro-facial; Non-odontogenic; Periapical radiolucency

Abbreviations: CECT: Contrast Enhanced Computed Tomography; IOPA: Intraoral Periapical Radiograph.

\section{Introduction}

Pain in oro-facial region is complex and distressing condition which frequently overlaps with various surgical and medical disciplines [1-7]. Accurate diagnosis could be challenging for any experienced clinician and Nonodontogenic toothache is one such entity. A misdiagnosis can lead to unnecessary treatment and may also exacerbate the symptoms for which the patient sought treatment. Osteomyelitis is one such entity having radiographic similarity with periapical radiolucency during its initial stage. Often such situation can lead to inaccurate diagnosis during dormant stage. Though due to advancement in medicine the incidence of osteomyelitis had a great decline still it can be an enigma to an endodontist in diagnosing the condition. Reported here is a case which highlights the role of endodontist while encountering and diagnosing a case of osteomyelitis.

\section{Case Report}

A 64 year old male patient who is farmer by occupation reported to the Department with the chief complaint of pain and swelling in right upper back tooth region since 2 months. The pain was gradual in onset, dull aching, intermittent, aggravated on mastication and relieved on analgesics. The patient had a history of fronto-maxillary sinusitis for which he was admitted to associate hospital and was referred to the dental hospital for pain as mentioned above. With positive history on diabetes type II, patient was on insulin therapy since 4 years. Past dental history revealed local 
treatment with drainage of pus, and consumption of systemic antibiotics but symptoms were persisting. Extraoral clinical examination was normal. On palpation, mild tenderness was present over right side upper lip region.

Intraoral examination revealed intact dentition with no carious tooth. Grade I mobility, mild tenderness, intraoral sinus with muddy yellow pus discharge was seen irt 14 and the volume increased on applying pressure. The Pocket dept mid root buccally in relation with 14 was $10 \mathrm{~mm}$. Electric pulp test revealed delayed response suggestive of irreversible changes IOPA irt 14 was inconclusive, hence final diagnosis of concomitant endo-perio with communication formulated. Treatment for 14-root canal treatment was decided.

Access opening was done with Endo Access bur and biomechanical preparation for Buccal and Palatal canals were completed in one visit. Working length-buccal $18 \mathrm{~mm}$ palatal $19 \mathrm{~mm}$ preparation was done upto30- 4\%(Neoendo) Calcium hydroxide(D-tech) intra-canal medicament was placed and patient was recalled after 1 week.

Patient reported after 15 days with multiple swellings and draining sinus from 14 to 24 region. Suspecting it to be underlying bony pathology, patient was referred to the Department of Oral and Maxillo-facial Surgery. OPG was advised but finding were inconclusive regarding osteomyelitis except ill-defined apical radiolucency irt with 14,15 . Swab was taken for pus culture. Systemic Antibiotic and analgesics were prescribed. (Augmentin 625mg TDS+ Metrogyl $400 \mathrm{mg}$ TDS+ Rantac 150mg + Zerodol P BD). Patient was kept on insulin chart at $6 \mathrm{hr}$ interval along for next5 days and advised for insulin therapy.

a) Postprandial blood sugar level- $160 \mathrm{mg} / \mathrm{dl}$

b) Random blood sugar level-140 mg/dl

c) Heamogram profile-

d) $\quad \mathrm{Hb}-10.2 \mathrm{gm} \%$

e) Total wbc-7900 cell/cumm

f) M.C.H-25.6pg

g) M.C.H.C- $31.8 \%$

h) While LFT and serum bilirubin levels were normal.

Pus culture report obtained after 3 days was inconclusive as it stated "multiple organisms can be isolated". Patient was recalled for further treatment 1 week later, however, the severity of symptoms and disease worsened. Patient reported within 5 days with complain of teeth mobility, Grade II mobility was seen with 11, 12, 14, 21, 22, 23 and Grade I mobility with $13,15,24,25$. Entire anterior maxillary segment was mobile. Considering it to be a fast spreading underlying bony pathology further, patient was referred for Contrast Enhanced Computed Tomography (CECT) by the Department of Oral and Maxillofacial Surgery (Figure 1).

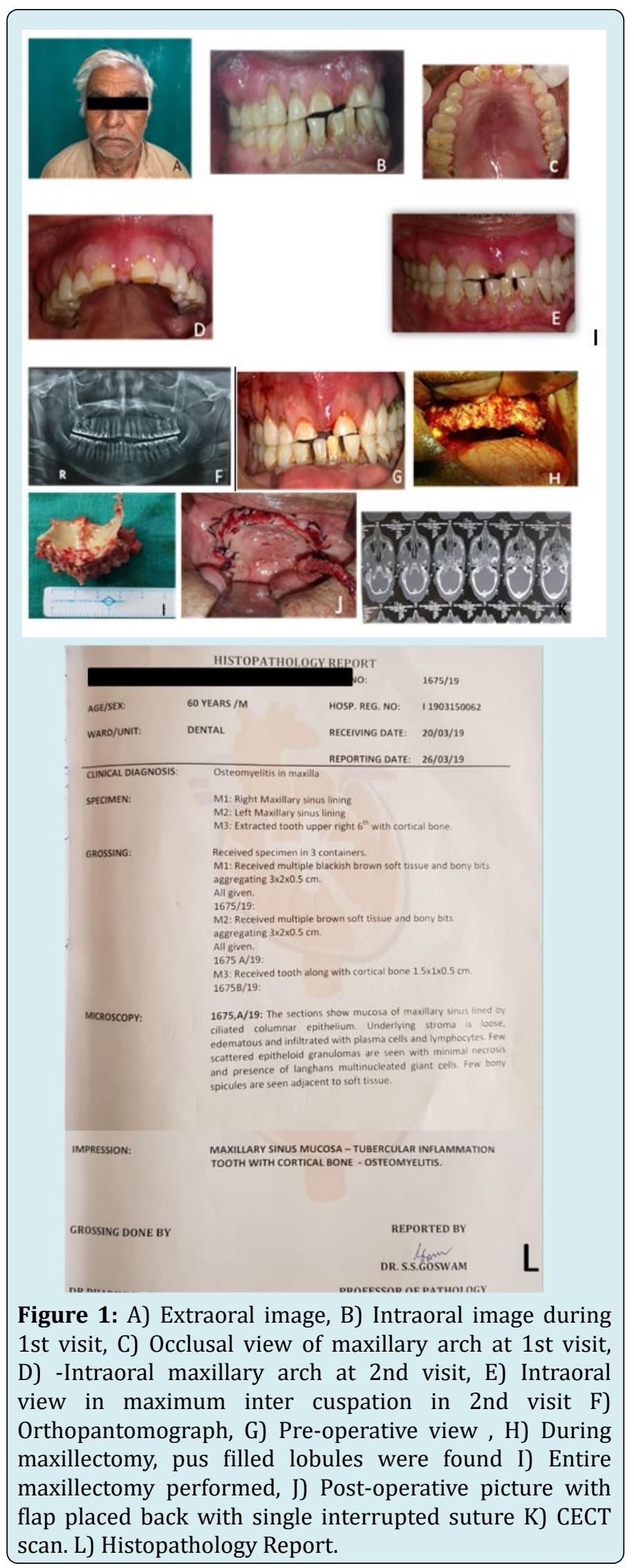




\section{Open Access Journal of Dental Sciences}

CECT showed the presence of extensive areas of bony destruction on maxillary sinus wall on the right and left side, alveolar process of maxilla, and orbital floor. Provisional diagnosis of osteomyelitis involving maxilla was made. Squamous cell carcinoma of the maxilla and fibrous dysplasia were included in the differential diagnosis and ruled out by clinical and radiological findings. A clinic-radiographic diagnosis of acute suppurative osteomyelitis involving maxilla was formulated, and the patient was taken up for maxillectomy by oral surgery department.

Maxillectomy was performed as per clinical extent of the pathology along with complete debridement of bone. Yellowish colored pus-filled lobules were seen during intraoperative procedure, complete debridement of bone was done and specimens were sent for the histo-pathological examination. Post-operative histo-pathological examination revealed-maxillary sinuslining with-tubercular inflammation and bony trabeculae with ragged margins. Patient is planned for further rehabilitation by ridge augmentation and full mouth prosthesis after complete recovery.

\section{Discussion}

As an endodontist it is very important for us to differentiate between Odontogenic and non odontogenic pathology. Most of the non odontogenic pathology also imitates pulpal periodontal lesion. Mis-diagnosis lead to inadvertent treatment ending in irreversible damage to dental tissue. Early diagnosis of aggressive pathology is important. It is rare to come across such conditions and hence the diagnosis becomes a race against time. Osteomyelitis of the maxillofacial region is uncommon with mandible is softer target than the maxilla [6,7]. The primary reason is a complex network of blood supply of maxilla is richer and thin cortical bone present [8].

In the present situation patient reported to an endodontist with history of chronic sinusitis with poor oral hygiene, pus discharge with deep pockets along with the delayed pulpal response, the reason was thought to be the odontogenic one, the final diagnosis as primary periodontal secondary endodontic lesion was done. As a first line of treatment root canal was performed. While evaluating for persistent pain, sudden development of multiple radiolucent areas over a period of two weeks with systemic history of diabetic mellitus, and multiple gingival swelling with pus drainage seen. We realized the condition has bony pathology rather than local tooth involvement hence the reference was done to the Dept of Oral and Maxillofacial Surgery. Absence of typical clinical findings in the present case like Deep Pain, High Intermittent Fever, Paresthesia or Anesthesia of Lower Lip, Pus discharge from the gingival crevice lead to delayed final diagnosis [9]. Constitutional signs of acute infection such as body ache, malaise, raised ESR, etc were also not evident in this case. Routine laboratory tests are usually nonspecific. The white blood cell count is often normal even in the setting of acute osteomyelitis. The gold standard for the diagnosis of osteomyelitis is bone biopsy with histopathologic examination and tissue culture [10]. Patient being a farmer and situated far from the hospital also contributed to the delay as he couldn't report early after first visit.

Typical radiographic changes always plays an important role for the clinician as in osteomyelitis usually demonstrate a "moth-eaten" appearance due to enlargement of medullary spaces and widening of Volkmann's canals resulting from destruction and replacement with granulation tissue The above mentioned case had misdiagnosed as sinusitis and odontogenic pain due to underlying bone pathology in its dormant stage and findings were not observed on OPG [5].

Poor periodontal condition, which leads to breakdown of the periodontal ligament facilitating deep invasion of pathogens, seems to be an important condition leading to osteomyelitis. Significant periodontal disease was found in $51 \%$ of the OM patients in a retrospective study [4]. CECT was advised as it provides excellent anatomic delineation of the infected area and surrounding soft tissue, assisting surgeon in avoiding morbidity and damage to adjacent critical structures. The role of an endodontist here was an meticulous examination with analyzing the clinical features which include deteriorating gingival condition, increase in bone destruction leading to mobility. Therefore, it is imperative that prompt diagnosis is to be made and aggressive treatment is initiated to avoid subsequent dreaded consequences.

\section{Conclusion}

Maxillary osteomyelitis is one of the most difficult to treat infectious disease, clinical suspicion is critical to initiate prompt and appropriate hematological, histological and radiological investigation. Aggressive medical management with adequate surgical intervention is the key to successful management. In the present case as an endodontist we could not save the tooth but definitely we could save the patient's life.

\section{References}

1. Alberts I (2008) Idiopathic Orofacial Pain: A Review. The Internet Journal of Pain, Symptom Control and Palliative Care 6(2).

2. Gupta V, Singh I, Goyal S, Kumar M, Singh A, et al. (2017) Osteomyelitis of maxilla-a rare presentation: case report and review of literature. Int J Otorhinolaryngol Head Neck Surg 3(3): 771-776. 
3. Nezafati S, Ghavimi MA, Yavari AS (2009) Localized Osteomyelitis of the Mandible Secondary to Dental Treatment: Report of a Case. J Dent Res Dent Clin Dent Prospects 3(2): 67-69.

4. Baltensperger MM (2003) A retrospective analysis of 290 osteomyelitis cases treated in the past 30 years at the Department of Cranio-Maxillofacial Surgery Zurich with special recognition of the classification (Doctoral dissertation).

5. Tsukayama DT (1999) Pathophysiology of posttraumatic osteomyelitis. Clin Orthopaed Rel Res 360: 22-29.

6. Baltensperger MM, Eyrich GK Osteomyelitis of the Jaws.
New York: Springer publications, pp: 5-50.

7. Flemming H (1925) Osteomyelitis of the skull. Cal West Med 23(8): 985-988.

8. Hudson JW (1993) Osteomyelitis of the jaws: a 50-year perspective. J Oral Maxillofacial Surg 51(12): 1294-1301.

9. Sujatha S Reddy, Kavitha P, Prashanthi C, Pallavi C, Poornima E (2014) Osteomyelitis of the Maxilla: A Case report of three cases. Am J Adv Med Sci 2(3): 34-41.

10. Fritz JM, McDonald JR (2008) Osteomyelitis: approach to diagnosis and treatment. Phys Sportsmed 36(1): 116823. 